\title{
Object detection in the fly during simulated translatory flight
}

\begin{abstract}
Translatory movement of an animal in its environment induces optic flow that contains information about the three-dimensional layout of the surroundings: as a rule, images of objects that are closer to the animal move faster across the retina than those of more distant objects. Such relative motion cues are used by flies to detect objects in front of a structured background. We confronted flying flies, tethered to a torque meter, with front-to-back motion of patterns displayed on two CRT screens, thereby simulating translatory motion of the background as experienced by an animal during straight flight. The torque meter measured the instantaneous turning responses of the fly around its vertical body axis. During short time intervals, object motion was superimposed on background pattern motion. The average turning response towards such an object depends on both object and background velocity in a characteristic way: (1) in order to elicit significant responses object motion has to be faster than background motion; (2) background motion within a certain range of velocities improves object detection. These properties can be interpreted as adaptations to situations as they occur in natural free flight. We confirmed that the measured responses were mediated mainly by a control system specialized for the detection of objects rather than by the compensatory optomotor system responsible for course stabilization.
\end{abstract}

B. Kimmerle $(\bowtie) \cdot A .-K$. Warzecha $\cdot M$. Egelhaaf Centre for Visual Sciences,

Research School of Biological Sciences,

Australian National University,

Canberra A.C.T. 2601,

Australia

Present address of all authors:

Lehrstuhl für Neurobiologie,

Fakultät für Biologie,

Universität Bielefeld,

Postfach 100131 ,

D-33501 Bielefeld, Germany,

Fax: +49-521/1066038;

e-mail: bernd.kimmerle@biologie.uni-bielefeld.de
Key words Figure-ground discrimination - Relative motion - Object detection - Insect visual system - Optic flow

Abbreviations $O M$ object motion $\cdot r B M$ rotatory background motion $t B M$ translatory background motion - TFO temporal frequency of the moving pattern defined as "object" - TFB temporal frequency of the moving pattern defined as "background"

\section{Introduction}

In order to navigate safely in their environment animals have to gather information about the structure of their surroundings. This implies that objects that might interfere with the path of locomotion (either as targets or obstacles) have to be discriminated from their background. Motion cues can play an important role in object detection (Helmholtz 1866). An object is seen more readily if it is moving relative to its background. Relative motion on the observer's retina can be elicited by a moving object as well as by a stationary object if the observer is in motion. During translatory self-motion the retinal image velocity of a stationary object depends on its angular position with respect to the observer's direction of motion as well as on its distance from the observer. A closer object passes by more quickly than does the background. Relative motion induced by the self-motion of the observer is, thus, also suited to provide a basis for depth vision (review: Collett and Harkness 1982). Many animals ranging from insects to primates can use relative motion cues to detect objects in front of their background (Braddick 1974; Miles and Kawano 1987; Srinivasan 1993). In particular flies (Kimmerle et al. 1996) as well as bees (Srinivasan et al. 1990) and hawkmoths (Pfaff and Varjú 1991) were shown to be able to use relative motion information that is elicited by their own locomotion to detect stationary objects elevated above the ground. 
A number of investigations at both the behavioural and the cellular level have been aimed at unravelling the mechanisms which enable the fly to discriminate objects from their background. In behavioural studies the turning responses of tethered flying flies towards objects defined solely by relative motion cues have been characterized in great detail (Virsik and Reichardt 1976; Reichardt and Poggio 1979; Reichardt et al. 1983; Egelhaaf 1985a; Egelhaaf. et al. 1988). Electrophysiological experiments have revealed a group of cells in the third visual neuropil of the fly, the so-called "figuredetection cells" (FD cells), which respond most vigorously when small objects move relative to their background in the frontolateral visual field (Egelhaaf 1985b). These cells are supposed to play a central role in mediating object-directed turning responses of the fly. In all these studies the background against which the object had to be discriminated was either stationary or was rotated around the vertical body axis of the animal, mimicking the retinal pattern motion a fly experiences when turning around this axis. Since in a free-flight situation, however, flies usually turn and translate at the same time, retinal image motion is in most cases composed of rotatory as well as translatory components. In the present study we therefore analyzed the reactions of tethered flying flies to objects defined by relative motion while simulating a straight forward flight of the animals. Translation was simulated by presenting front-to-back pattern motion in front of both eyes. We investigated how the detectability of an object as judged by the average strength of the fly's turning response depends on retinal object velocity and retinal background velocity. Our results suggest that the performance of the flies seems to be well adapted to situations as encountered by these animals in natural free flight.

\section{Materials and methods}

The experiments were carried out with female blowflies Lucilia cuprina, which were raised in laboratory stocks of the C.S.I.R.O., Division of Entomology, Black Mountain Laboratories, Canberra, Australia.

The head of the animal was fixed to the thorax with wax either under light $\mathrm{CO}_{2}$ anesthesia or after cooling the animal down to a temperature of approximately $4{ }^{\circ} \mathrm{C}$. A triangular piece of cardboard was glued to the wax just above the pronotum. Newly prepared animals were allowed to recover for at least 1 day before they were used in an experiment. The cardboard triangle served to attach the fly to a torque compensator. This device measured the torque of the stationarily flying fly around its vertical body axis (Fermi and Reichardt 1963; Götz 1964). The torque signal was fed into the computer at a sampling rate of $100 \mathrm{~Hz}$ via an I/O Card (Data Translation, DT2801-A) and stored for further analysis. The stimuli were presented on two CRT screens (Tektronix 608), which were placed symmetrically in front of the fly subtending an angle of $90^{\circ}$ (Fig. 1A). The fly was positioned at the point where the orthogonals through the screen centres intersect, its body axis pointing towards the midline between the two monitors. In this configuration the right (left) screen subtended a horizontal visual angle from $+(-) 11^{\circ}$ to $+(-) 80^{\circ}$ in front of the right (left) eye with respect to the longitudinal body axis. In the vertical the screens extended from $+29^{\circ}$ (dorsal visual field) to $-29^{\circ}$ (ventral visual

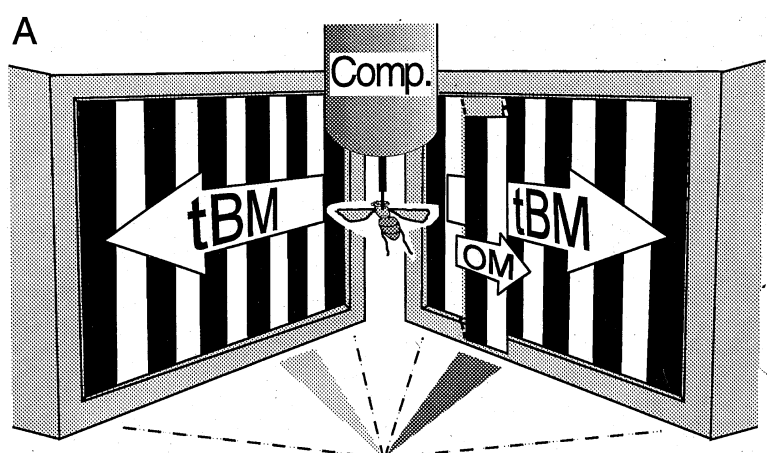

B

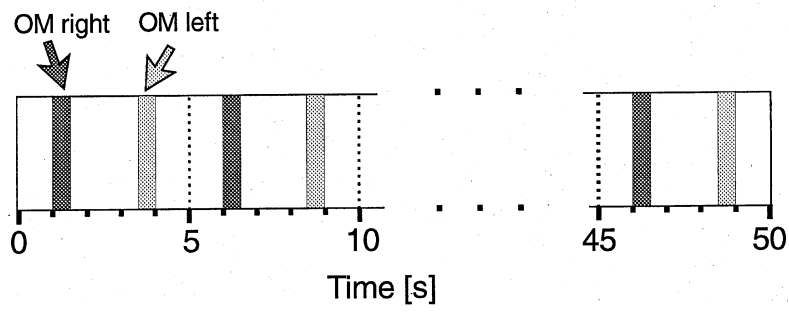

Fig. 1 A Schematic drawing of the experimental setup. The fly was fixed to a torque compensator (Comp.) and positioned in front of two monitors, the body axis pointing towards the midline between both monitors (horizontal visual angle: $0^{\circ}$ ). A periodic square wave grating could be moved from front to back ( $t B M$ : translatory background motion) at different temporal frequencies. Part of the right screen is shown popped out in order to demonstrate that the pattern within this object window could be moved independently of the remaining parts of the screen (OM: object motion). The respective object window on the left screen is not shown popped out because object motion was never presented simultaneously on both screens. The horizontal extent of the (right/left) object windows is indicated by the (dark/light) shaded areas, the horizontal extent of the background by the dashed lines. B Time-course of a single stimulus presentation. It lasted $50 \mathrm{~s}$ and consisted of coherent front-to-back motion of all pattern elements on both screens (white areas), interrupted by 20 object motion intervals of $0.5 \mathrm{~s}$ duration (shaded bars). Object motion stimuli were presented in turns on the right (dark bars) and on the left (light bars) screen with inter-stimulus intervals of $2 \mathrm{~s}$ duration. Consequently, one such presentation can be decomposed in ten identical components as indicated by the dotted lines

field). Periodic square wave gratings were generated by two image synthesizers (Innisfree, Picasso) at a frame rate of $100 \mathrm{~Hz}$. The luminance of the bright and dark stripes was in the range of $35-40 \mathrm{~cd} \cdot \mathrm{m}^{-2}$ and $3.5-4 \mathrm{~cd} \cdot \mathrm{m}^{-2}$, respectively, yielding a contrast of approximately 0.8 on each screen. The spatial wavelength of the pattern amounted to $1.1 \mathrm{~cm}$, corresponding to a visual angle of about $6.3^{\circ}$ in the region where the object was displayed. On both screens the pattern was moved from front to back, in this way simulating translatory background motion (tBM) as experienced by the animal during straight flight. Within a window on each screen extending from $+(-) 19.0^{\circ}$ to $+(-) 31.6^{\circ}$ in the horizontal and across the whole screen in the vertical direction the pattern could be moved independently from the remaining part of the screen in order to mimick the presence of an object. Relative motion was introduced by changing the temporal frequency of the pattern within one of the object windows ("object motion", OM) with respect to the background. Note that the term "object motion" does not imply that the object window itself is moving but rather the stripe pattern within its boundaries. Like background motion, object motion was always directed from front to back. There was usually a phase shift between the pattern within the object window and the pattern within the rest of the screen. This phase shift at the edges of the object windows enabled a human observer to detect these windows even when the pattern within 
them moved at the same temporal frequency as in the remaining parts of the screen; however, it is very unlikely that flies detect an object under these conditions. Previous experiments have shown that only differences in velocity between object and background and not differences in texture lead to the detection of an object by the fly (Virsik and Reichardt 1976). In these experiments not even a black bar could be detected in front of a randomly textured background as long as it was moving coherently with the background.

As an indicator for the detection of object motion, the turning responses of flies towards these virtual objects were recorded using 42 different stimulus conditions. These represented all possible combinations of seven different temporal frequencies for object motion (TFO) and background motion (TFB) $(0,0.5,1,2,4,8$, $16 \mathrm{~Hz}$ ) excluding the seven combinations yielding no relative motion (i.e. same temporal frequencies of object and background motion). These 42 stimulus conditions were presented in random order, each one 28 times to a group of 44 flies. One presentation lasted $50 \mathrm{~s}$, the time-course of which is schematically depicted in Fig. 1B. Each presentation started with translatory background motion, the temporal frequency within the object windows being the same as in the remaining parts of the screens. During intervals of $0.5 \mathrm{~s}$ the temporal frequency within one of the object windows was changed, thus leading to relative motion between object and background. Object motion was presented in turns on the left and on the right screen, always interrupted by $2 \mathrm{~s}$ of coherent translatory pattern movement, resulting in ten single object motion stimuli per presentation on each side. Hence, for each of the 42 stimulus conditions a total of $28 \times 10 \times 2=560$ responses were recorded. In about half of the presentations the initial object motion stimulus was presented on the left screen; in the other half it was presented on the right screen. The strength of each turning response was calculated by taking the average torque during the 0.5 -s object motion interval and subtracting the average torque during the $0.5-\mathrm{s}$ interval prior to the onset of object motion. These response values were averaged after sign-inverting the response values obtained during object motion on the left screen. Torques to the left yielded negative values, torques to the right yielded positive values.

In further experiments an additional set of stimulus conditions was presented with an identical time-course as in the preceding experiment. Instead of the object motion stimuli within the $0.5-\mathrm{s}$ intervals the whole pattern on both screens was coherently moved in turns clockwise and counterclockwise, thereby simulating the retinal image displacements a fly experiences when rotating around its vertical body axis to the left and to the right, respectively ("rotatory background motion", rBM). During the 2-s intervals between rotations the pattern was stationary. The responses obtained in these experiments were compared with the responses to object motion in front of a stationary background, i.e. a subset of stimulus conditions as used in the previous experiment. For this purpose the responses to 21 presentations of six OM and six rBM conditions were recorded. These experiments were conducted with a group of 15 flies. The reactions were evaluated in the same way as before.

\section{Results}

During simulated translatory flight flies are able to detect objects solely defined by relative motion and respond with an intended turn towards them. The strength of the average turning response depends on the TFO and TFB. Figure 2 shows two examples of average turning responses to object motion stimuli with different combinations of TFO and TFB. The response traces illustrate the time-course of the average torque generated by the flies during translatory pattern motion in the absence of object motion and during object motion on the right and on the left screen. After the onset of object motion a

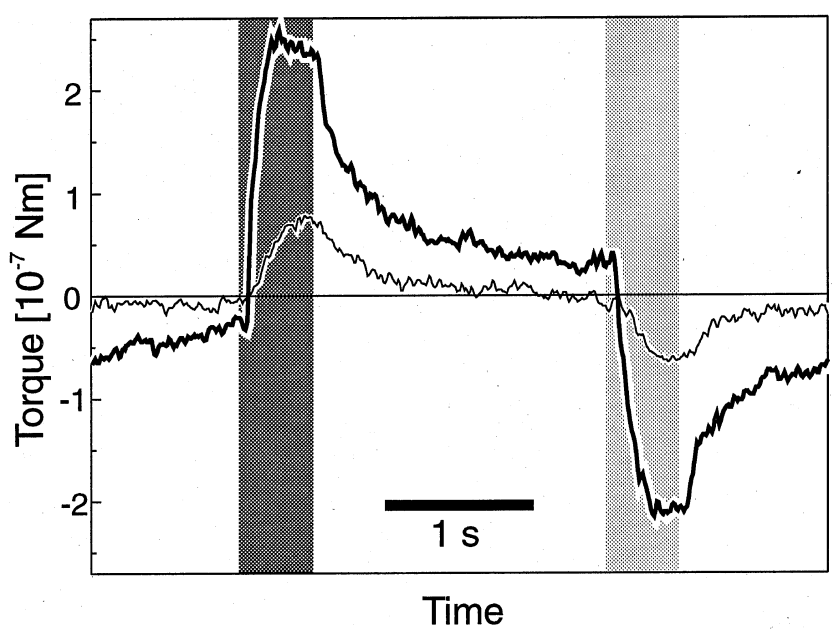

Fig. 2 Time-course of two average torque traces showing the turning responses to virtual objects defined by relative motion on the right (dark shaded area) and on the left (light shaded area) screen. During the remaining time (white area) no relative motion and hence no object was present. Positive values indicate a torque to the right, negative values a torque to the left. The 0 -torque level was determined separately for each stimulus presentation. By definition, it was chosen to be the average torque during 20 time intervals, each of $0.5 \mathrm{~s}$ duration, just prior to the onset of object motion. The average torque traces of two stimulus conditions are shown (each trace represents the average of 280 individual responses). Thick trace: TFO: $16 \mathrm{~Hz}$, TFB: $2 \mathrm{~Hz}$. Thin trace: TFO: $4 \mathrm{~Hz}$, TFB: $2 \mathrm{~Hz}$. Shortly after the onset of object motion the fly tends to turn towards the location of the virtual object. After cessation of object motion, i.e. during coherent translatory background motion, the torque gradually approaches the 0-level. Flies turn more strongly towards the faster moving object

sudden change in the torque generated by the flies indicates the intention of the animals to turn towards the object. The thin trace shows the turning response to object motion at a temporal frequency of $4 \mathrm{~Hz}$, the thick trace to object motion at a temporal frequency of $16 \mathrm{~Hz}$. In both cases the background pattern moved at a temporal frequency of $2 \mathrm{~Hz}$. Object motion at a higher temporal frequency thus elicits a stronger torque response. As soon as object motion ceases, the torque gradually returns towards the zero level. If the response is strong this might not be accomplished completely within the period between successive object motion stimuli (see thick trace).

The dependence of the turning response on the temporal frequency of object motion is shown in Fig. 3 for two TFBs $(0 \mathrm{~Hz}$ and $2 \mathrm{~Hz})$. When the background is stationary the responses increase with increasing TFO up to a temporal frequency of $8 \mathrm{~Hz}$ (Fig. 3A). When the background is moving at a temporal frequency of $2 \mathrm{~Hz}$ (Fig. 3B) there are hardly any object-directed reactions of the flies as long as object motion is slower than background motion. Conversely, when object motion is faster than background motion the response amplitude increases with increasing TFO up to the highest temporal frequency tested $(16 \mathrm{~Hz})$. The necessity of TFO exceeding TFB in order to elicit clear turning responses can be observed for all TFBs used in our experiments (see below). 


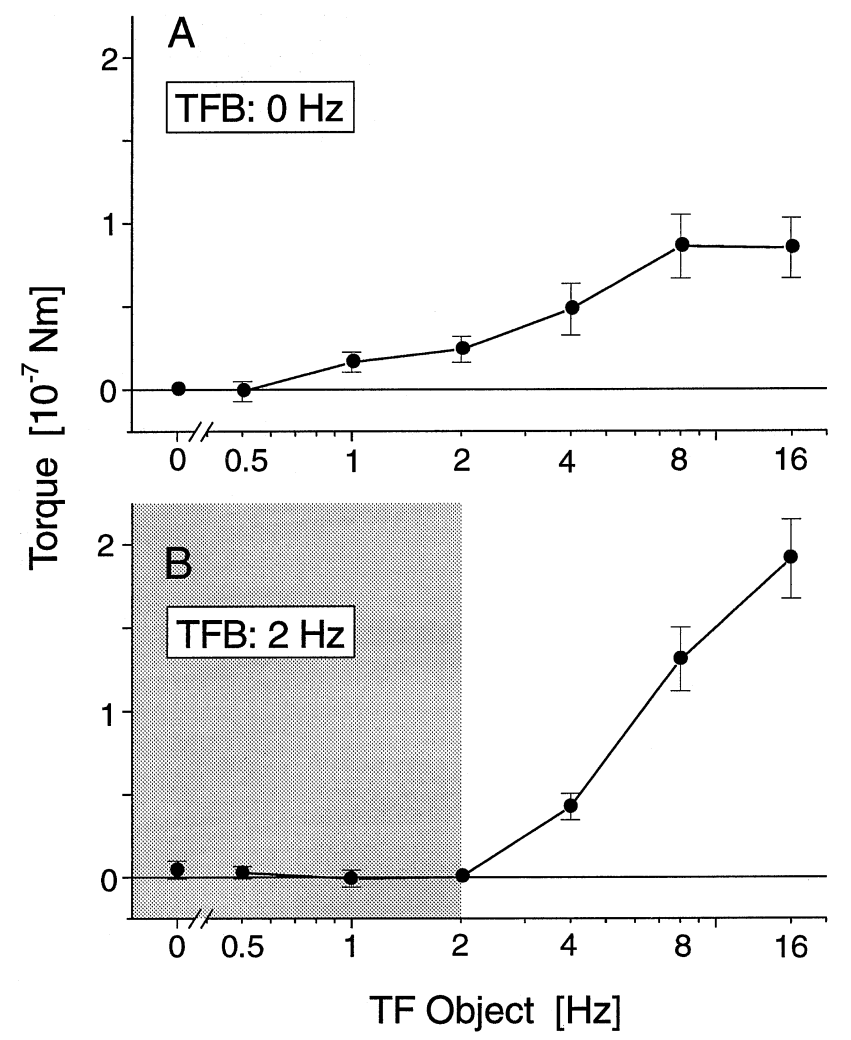

Fig. 3A, B Dependence of the turning response on object velocity: shown are the mean responses obtained for six stimulus conditions of object motion with a stationary background (A) and with background motion at a temporal frequency of $2 \mathrm{~Hz}(\mathbf{B})$. The two data points representing combinations that would not result in relative motion (TFO: $0 \mathrm{~Hz}$, TFB: $0 \mathrm{~Hz}$ and TFO: $2 \mathrm{~Hz}$, TFB: $2 \mathrm{~Hz}$ ) were assigned the value 0 . All other data points represent mean torque values of 560 individual responses obtained in 28 presentations. Error bars denote SEM $(n=28)$. The combinations with TFB exceeding TFO are shaded. The amplitude of the turning responses increases with increasing object velocity as long as the object moves faster than the background

Comparing the turning responses to fast object motion at different TFBs reveals another interesting feature of the performance of the fly in object detection (Fig. 4): across a wide range of temporal frequencies background motion enhances the turning response towards the object as compared to the response when the background is stationary. The response to object motion at a temporal frequency of $16 \mathrm{~Hz}$ is maximal during translatory background motion at $2 \mathrm{~Hz}$. With respect to the stationary background condition the response increases, on average, about twofold. Responses to object motion at lower temporal frequencies (e.g. $8 \mathrm{~Hz}$, data not shown) exhibit similar characteristics, i.e. maximal responses during background motion at $1-2 \mathrm{~Hz}$, suggesting that object detection on the basis of relative motion is best if the background is not stationary but moves across the retina at a moderate temporal frequency.

The set of responses is summarized in a contour plot (Fig. 5). Due to the logarithmic scaling the combinations with either stationary background or stationary object (TFO or TFB: $0 \mathrm{~Hz}$ ) are omitted. The degree of

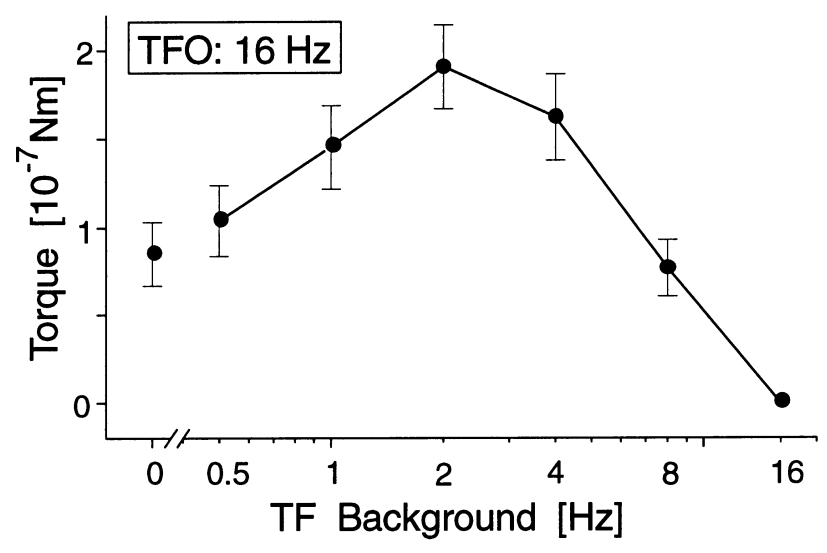

Fig. 4 Dependence of the turning response on background velocity. Shown are the mean responses to object motion at a temporal frequency of $16 \mathrm{~Hz}$ obtained for six TFBs. The datapoint representing the combination that would not result in relative motion (TFO: $16 \mathrm{~Hz}$, TFB: $16 \mathrm{~Hz}$ ) was assigned the value 0 . All other data points represent mean torque values of 560 individual responses obtained in 28 presentations. Error bars denote SEM $(n=28)$. The amplitude of the turning response is maximal when the background moves at a temporal frequency of $2 \mathrm{~Hz}$. As compared to a stationary background, translatory background motion can enhance the turning response by a factor of approximately 2

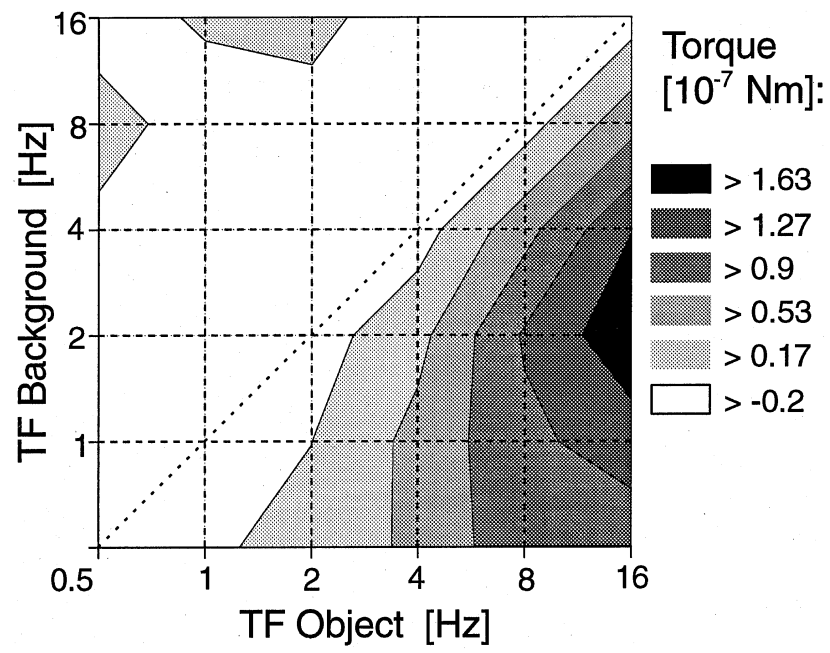

Fig. 5 Contour plot of the turning responses for all combinations of temporal frequencies of object motion and translatory background motion with the exception of those in which either the background or the object was stationary (owing to logarithmic scaling). Intersection points of the dashed lines represent the conditions for which data points were obtained; the contour lines (solid) are interpolated. The data points along the dotted diagonal from the lower left to the upper right of the plot, i.e. the combinations with TFO $=\mathrm{TFB}$, were not measured but assigned the value 0 . All other data points represent mean torque values of 560 individual responses obtained in 28 presentations. Flies show strong turning responses when TFO $>$ TFB (combinations right of the diagonal) and react only weakly when TFB $>$ TFO (combinations left of the diagonal)

shading codes for the response strength under the respective stimulus conditions, the dark areas indicating strong turning responses towards the object. When TFB exceeds TFO either no turning responses are elicited at 
all or only weak ones, the latter preferably when the background is moving considerably faster than the object (upper left corner). On the other hand, when TFO exceeds TFB the flies respond with strong turns towards the object. The slopes along the horizontals, i.e. the increase of the response owing to increasing TFO at a given TFB, becomes steeper with increasing TFB. Therefore, fast moving objects can be detected even in the presence of translatory background motion at considerable temporal frequencies. The response peak lies at the outer rim of the plot, i.e. the maximal torque amplitude was achieved with the highest TFO $(16 \mathrm{~Hz})$ employed in the experiments. Even stronger responses might have been elicited with TFOs exceeding this value.

Turning responses of the fly around its vertical body axis are mediated by at least two different control systems. The compensatory optomotor system is most sensitive to retinal motion as elicited by rotations of the animal whereas the object detection system is most sensitive to small moving objects (see Discussion). Is it possible to assign the responses described in the present account to one of these systems? If the turning responses were mediated by the compensatory optomotor system as a consequence of a motion imbalance during the periods of object motion, then the optimal optomotor stimulus, i.e. horizontal background motion on both screens mimicking rotation of the fly around its vertical body axis should lead to stronger or at least equally strong responses as object motion. To test whether the compensatory optomotor system is involved in mediating the described turning responses we conducted another set of experiments, replacing object motion by rotatory background motion. The 0.5 -s intervals during which the whole pattern on both screens was moved successively clockwise and counterclockwise were interrupted by 2-s intervals during which the pattern was stationary. The resulting responses are compared with responses to object motion with a stationary background (same conditions as in Fig. 3A). Figure 6A shows the time-dependent responses to object motion at a temporal frequency of $8 \mathrm{~Hz}$ with a stationary background and to rotatory background motion at a temporal frequency of $8 \mathrm{~Hz}$. This comparison reveals that the torque increases more rapidly and reaches a plateau more quickly when the fly is turning towards a virtual object than when it is trying to compensate for largefield pattern motion. At all tested temporal frequencies the object-directed turning responses exceeded the responses to rotatory background motion (Fig. 6B). Since the response of the optomotor system is known to increase with pattern size, but in the present experiments object motion elicits stronger responses than rotatory background motion, we conclude that the compensatory optomotor system plays only a minor role in mediating turning responses towards small objects as observed in the present study. Thus, the main response component is mediated by a control system most sensitive to small moving objects.

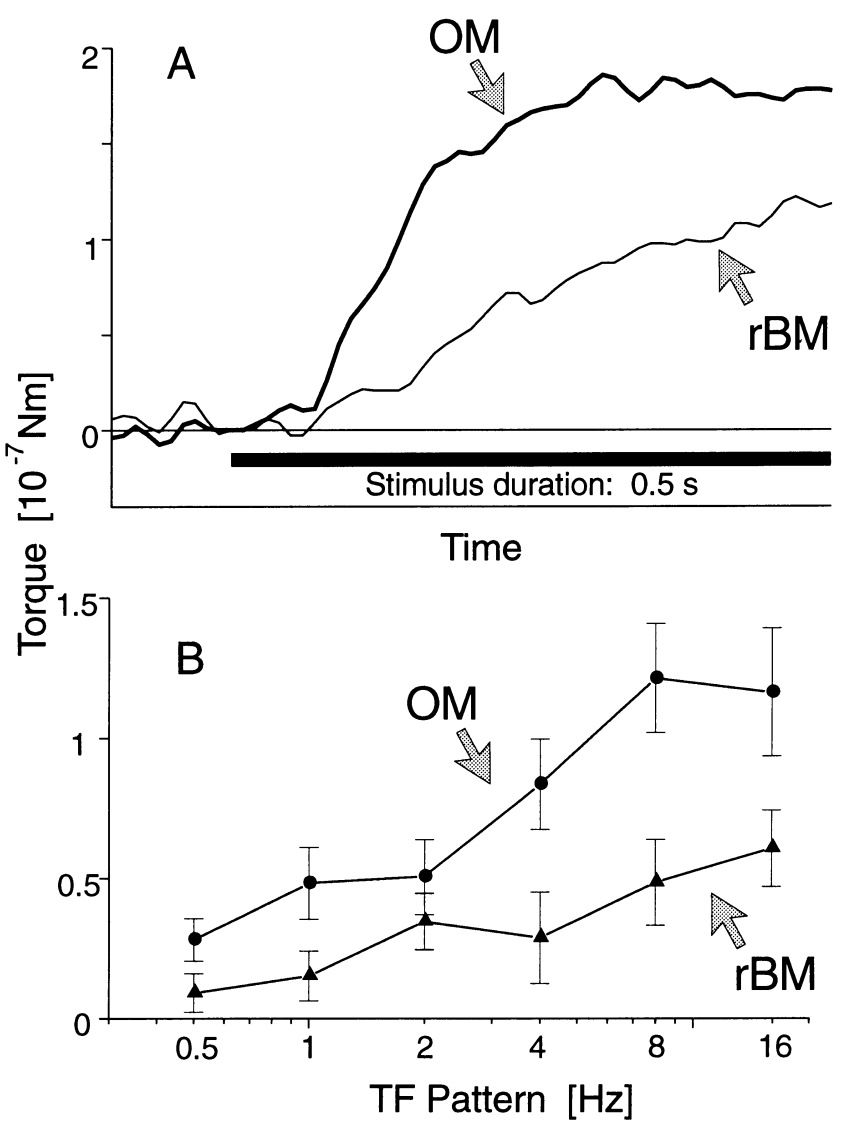

Fig. 6A, B Comparison of the turning responses to object motion and rotatory background motion: A time-course of the average torque elicited during large-field rotatory background motion on both screens $(r B M)$ and during small-field object motion $(O M)$. Each torque trace represents an average of 210 individual responses obtained in 21 presentations. The temporal frequency of pattern motion amounted to $8 \mathrm{~Hz}$ in both cases. Before stimulus onset the pattern was stationary. To allow for better comparison of the time-course both curves were shifted along the torque axis by an amount that resulted in 0 torque at stimulus onset. OM leads to a steeper increase in torque generation than $\mathrm{rBM}$, thus indicating that the torque responses elicited by the two different stimulus conditions cannot be mediated by the same control system; B comparison of the responses at different pattern velocities. The time-course of the presentations was as in Fig. 1b. In the conditions with object motion (OM) the background was stationary; in the conditions with rotatory background motion (rBM) the pattern was stationary during the inter-stimulus intervals. Data points represent mean values of 420 individual responses obtained in 21 presentations. Error bars denote SEM $(n=21)$. Irrespective of pattern velocity, the turning response to small objects exceeds the compensatory optomotor response elicited by large field rotation at the same velocity

\section{Discussion}

During simulated translatory flight, flies try to turn towards virtual objects defined by relative motion between object and background. The turning response depends in a characteristic way on the velocity of both object and background motion. Object motion at increasing velocities leads to stronger responses only as long as the velocity of object motion exceeds that of background motion, otherwise no distinct responses are elicited. 
Background motion within a certain range of velocities seems to have a facilitatory effect on object-directed turns. The turning responses to object motion are stronger than to rotatory large-field motion around the vertical body axis of the animal and are thus not mediated by the optomotor system for course stabilization.

\section{Limitations in simulating a translatory flow field}

How well do the stimuli used in our presentations approximate a realistic flow field as experienced by a fly during translatory forward flight? In order to address this question we compare the retinal flow elicited by our stimuli with the retinal flow in free straight forward flight. In unrestrained flight the retinal motion vectors expand radially from the direction of heading. They are aligned horizontally along the equator and with increasing elevation of the observed object the inclination of the respective motion vector increases. The retinal velocity of a given point in space depends on its angular deviation from the heading pole of the observer as well as on the distance of this point from the observer. Owing to the influence of distance on retinal velocity there is an infinite number of possible distributions of retinal velocities, each corresponding to a specifically structured surround. In our presentations the stripe patterns were moving in the horizontal direction over the whole extent of the screens. The temporal frequency of translatory background motion was constant on both screens. Owing to perspective distortion the angular velocity of the pattern depended on the visual angle. Hence with respect to the alignment of the motion vectors our artificial flow field differs from a realistic one, whereas the distribution of norms of the motion vectors represents only a very special case. Despite the mentioned differences our translatory stimuli have one essential feature in common with real translatory flow fields: motion is directed from front to back in front of both eyes. Stimuli as we used them proved to be effective in eliciting behavioural responses that control translatory flight such as the modulation of the thrust force in tethered flying fruitflies (Götz 1968), the induction of the landing behaviour in houseflies (Borst 1990) as well as compensatory translatory movements in hovering hawkmoths (Kern 1994).

Object detection in the behavioural

and neuronal context of flight control in flies

How does the object-directed turning behaviour of tethered flying flies during simulated translation fit into the framework of the present knowledge about flight control in flies and its neuronal substrate (review: Egelhaaf and Borst 1993)? The yaw torque is controlled by at least two parallel systems with different spatial and temporal properties (Egelhaaf 1987). According to their spatial properties the control systems were named "large-field system" and "small-field system". The for- mer is most sensitive to motion within large parts of the visual field as induced by deviations of the animal from a straight flight path and is assumed to control the compensatory optomotor response. The latter is suggested to control the turning responses towards objects moving in front of their background. With respect to their dynamic features the two systems differ in that the signals conveyed by the large-field system are low-pass-filtered resulting in a reduced sensitivity to fast changes in motion stimulation. Neural correlates for the large-field and the small-field system on the level of the lobula plate, the centre for the integration of motion information within the fly brain, are the three HS-cells ("horizontal system", Hausen 1982a,b) and the FD cells ("figure detection"; Egelhaaf 1985b), respectively. These cells collect local motion information on their extensive dendritic branchings and have large receptive fields. They are tuned to horizontal large-field motion (HS cells) and to horizontal motion of an object relative to its background (FD cells), respectively.

The control experiment (Fig. 6) provided evidence that the object-induced responses of the present experiments are mediated mainly by the small-field system. This conclusion is based on the following observations. (i) Comparing the time traces of the turning reactions reveals that the torque responses elicited by object motion increase at a faster rate than those elicited by rotatory background motion (Fig. 6A). This observation is in accordance with what would be predicted on the basis of the dynamic properties of the small-field and the large-field system, respectively. (ii) The averaged responses to small-field object motion exceed those to large-field rotatory background motion at corresponding temporal frequencies. Since the optimal stimulus for the compensatory optomotor system is large-field pattern motion the object-directed turning responses as measured in the present experiment cannot be dominated by the compensatory optomotor system but are mediated mainly by the small-field system.

Can our results be explained on the basis of the properties of the FD cells? The steady-state response of motion-sensitive cells in the lobula plate is maximal at temporal frequencies in the range $2-5 \mathrm{~Hz}$. This characteristic feature has been demonstrated in a variety of cells in the lobula plate (Eckert 1980; Hengstenberg 1982; Hausen 1982b; Maddess and Laughlin 1985) though not yet in FD cells. Since this response optimum is due to the characteristics of local input elements which can be assumed to be shared by all the integrating neurons in the lobula plate, it is most likely that FD cells exhibit the same properties in this respect. On this basis, it seems surprising that the behavioural responses to object motion superimposed on translatory background motion increase up to temporal frequencies of $16 \mathrm{~Hz}$. However, to account for the strong behavioural reactions at high temporal frequencies one has to consider the response dynamics of the neurons in the lobula plate. They depend differently on temporal frequency under transient and steady state conditions. Whereas the above-men- 
tioned optimum in the range $2-5 \mathrm{~Hz}$ refers to steadystate conditions, the response maximum of the cells under transient conditions is shifted towards higher temporal frequencies (Hausen 1982b; Maddess and Laughlin 1985; Egelhaaf and Borst 1989). Considering the short duration of object motion, this fits well with the behavioural result that objects are detected best at temporal frequencies as high as $16 \mathrm{~Hz}$.

FD cells are able to signal relative motion and thus to detect objects because their responses to motion within the receptive field are influenced by surround motion. In this respect, FD cells have similar characteristics to cells found in the cat (Sterling and Wickelgren 1969), in the privet hawkmoth (Collett 1971; Collett and King 1975), in the pigeon (Frost and Nakayama 1983; Frost et al. 1988) and in primates (Allman et al. 1985; Tanaka et al. 1986). Only two of the set of four FD cells are relevant in the present context: The FD1 and the FD4 cell are selective for front-to-back motion in front of the ipsilateral eye, i.e. their preferred direction coincides with the direction of pattern motion in the present experiments. Thus, they are suited to encode relative motion information as provided in these experiments. The neuronal circuitry responsible for tuning the FD1 cell to motion of small objects has been analyzed in great detail. Small-field tuning of the FD1 cell is due to inhibition mediated by the $\mathrm{VCH}$ cell, which is most sensitive to binocular rotatory large-field motion as elicited when the animal is turning around its vertical body axis (Warzecha et al. 1993; Egelhaaf et al. 1993; Borst and Egelhaaf 1993). Despite this knowledge, it is not possible to predict the responses of this neuronal circuitry to the stimuli used in the present experiments. To answer the question of whether the response properties of the FD cells can account for the object-directed turning behaviour of the fly during translatory background motion, electrophysiological recordings from both the FD1 cell and the FD4 cell have to be done using the same stimuli as in the behavioural experiments. Depending on the outcome of such investigations it might be possible to propose a straightforward link between object-directed turning behaviour and figure-ground discrimination at the level of output elements of the optic lobes. Alternatively, one might obtain hints on further processing stages involved in mediating turning responses towards small objects.

\section{Adaptations of object detection behaviour in the fly}

The most interesting observations of this study were: (i) that flies only respond to object motion if it is faster than background motion, and (ii) that background motion has a facilitatory effect on object-directed turning behaviour. These results can be interpreted in the light of their relevance in free-flight situations.

As long as the moving fly is not actually turning to fixate an object, the object's retinal velocity exceeds that of the more distant background. This is a common sit- uation when passing an elevated object in the flight path such as a flower. On the contrary, the object moving slower than the background would correspond to a situation with a "hole" in the background, a rather artificial case of presumably minor significance in a natural environment. Consequently, the system mediating object detection seems to be well tuned to detect stationary objects during flight. In contrast to stationary objects, the retinal velocity of a non-stationary object may be lower than that of the background. Consider, for example, a chase in which one fly is following another one, both flying in the same direction. Chasing behaviour in flies is mediated by a specific control system which is only present in males (Land and Collett 1974; Wehrhahn 1979; Wagner 1986). In this context, it should be noted that the present experiments were conducted with female flies only. Hence, the behavioural object-induced responses we examined probably play a minor role in the pursuit of conspecifics and rather serve the detection of stationary objects in the flight path.

There are parallels described in two other species that are reminiscent of the observation that flies almost exclusively respond to stimuli in which object velocity exceeds background velocity. In behavioural experiments bees were trained to land on edges defined by relative motion elicited in flight owing to height differences between the two surfaces forming the edge. Landing occurred frequently when bees approached the edge facing the elevated side but hardly ever when they approached the edge facing the lower side, indicating that the landings on the edges are triggered by a local increase rather than by a decrease in motion speed perceived at the edge (Lehrer and Srinivasan 1993). Frost et al. (1988) demonstrated that tectal cells in pigeons do not respond to random dot kinematograms in which an object solely defined by relative motion appeared as a "hole" in the background, whereas they strongly respond to an object that appeared as elevated above the background. However, in the case of the kinematograms the depth impression was determined by the direction of motion of the border of a group of coherently moving dots making up the object, whereas in our case relative depth was defined on the basis of the relative velocities of object and background. Consequently, the mechanisms mediating this neuronal specificity on the one hand and the fly's turning behaviour towards objects on the other hand must be different.

The significance of the facilitatory effect of background motion is straightforward to assess. The fly has to accomplish figure-ground discrimination during flight which implies, assuming that the background is structured and not at infinite distance, that the image of the background on the retina is not stationary but in motion. As this has been shown to be the condition under which the system mediating object detection works most efficiently, it may reflect yet another adaptation to the requirements in free flight. The dependence of the turning response on the velocity of translatory background motion shows a distinct optimum. Therefore, 
one might speculate that under free-flight conditions in a natural environment the performance in figure-ground discrimination is optimal when the flight speed of the fly lies within a certain range. It would be interesting to compare the range of optimal temporal frequencies as measured in the described experiments with an estimate about retinal background velocity during free flight of an animal in its natural habitat. However, this estimate is hard to obtain since the spectrum of temporal frequencies depends on the structure as well as on the distance of objects in the natural surroundings.

With respect to the facilitatory effect of background motion, it is interesting to compare the behavioural responses of the fly with properties of motion sensitive neurons in primate area MT. These cells are supposed to play a role in figure-ground discrimination since it was discovered that their responses to motion stimuli in the central receptive field are strongly influenced by an extensive antagonistic surround (Allman et al. 1985; Tanaka et al. 1986). A more recent study has demonstrated that surround inhibition in many MT cells is confined to restricted regions (Xiao et al. 1995), allowing for the extraction of complex features from the visual environment (Buracas and Albright 1996). Furthermore, it was shown that in addition to the class of MT cells with an antagonistic surround there exists a second class of cells whose response to motion in the preferred direction in the central receptive field is reinforced by surround motion in the same direction (Born and Tootell 1992). On the basis of our findings, one might speculate that cells with similar response properties as those described by Born and Tootell (1992) could underly object detection during translatory flight in flies. However, in all the cellular studies mentioned here the background was chosen to move in one direction, corresponding to situations when a stationary observer is tracking objects by eye or head movements. In contrast, in our experiments background motion was chosen to simulate translation of the fly.

In order to gain a deeper insight into the problems and limitations of object detection in free flight, it seems necessary to bring the experimental stimuli closer to the situation as encountered by a freely flying fly. The next step in this direction would be to leave the extreme cases of simulating pure rotation or pure translation and to combine these components in a realistic way. To do so, it is essential to analyse free flight behaviour and the optic flow a fly is subjected to in flight owing to its own actions and reactions.

Acknowledgements We want to thank Roland Kern, Volker Dürr and Dezsö Varjú for critically reading the manuscript, Judith Eikermann for skilful help in part of the experiments, Shao Wu Zhang for valuable suggestions and support in repairing and calibrating the torque meter, Karl Götz for the supply of spare parts for the torque meter, and the electronic and the mechanical workshops of the Research School of Biological Science for technical assistance. Bernd Kimmerle is especially indebted to Mandyam Srinivasan for financial support and encouragement during this work.

\section{References}

Allman J, Miezin F, McGuinness E (1985) Direction- and velocityspecific responses from beyond the classical receptive field in the middle temporal visual area (MT). Perception 14: 105-126

Born RT, Tootell RBH (1992) Segregation of global and local motion processing in primate middle temporal visual area. Nature 357: 497-499

Borst A (1990) How do flies land? From behavior to neuronal circuits. Bio Science 40: 292-299

Borst A, Egelhaaf M (1993) Processing of synaptic signals in fly visual interneurons selectively responsive to small moving objects. In: Aertsen A, Seelen W von (eds) Brain theory - spatiotemporal aspects of brain function. Elsevier, Amsterdam, pp 47-66

Braddick OJ (1974) A short-range process in apparent motion. Vision Res 14: 519-527

Buracas GT, Albright TD (1996) Contribution of area MT to perception of three-dimensional shape: a computational study. Vision Res 36: 869-887

Collett TS (1971) Visual neurones for tracking moving targets. Nature 232: 127-130

Collett TS, Harkness LIK (1982) Depth vision in animals. In: Ingle DJ, Goodale MA, Mansfield RJW (eds) Analysis of visual behaviour. MIT Press, Cambridge, MA, pp 111-176

Collett TS, King AJ (1975) Vision during flight. In: Horridge GA (ed) The compound eye and vision in insects. Claredon Press, Oxford, pp 437-466

Eckert H (1980) Functional properties of the H1-neurone in the third optic ganglion of the blowfly, Phaenicia. J Comp Physiol A 135: 29-39

Egelhaaf M (1985a) On the neuronal basis of figure-ground discrimination by relative motion in the visual system of the fly I. Behavioural constraints imposed on the neuronal network and the role of the optomotor system. Biol Cybern 52: 123-140

Egelhaaf M (1985b) On the neuronal basis of figure-ground discrimination by relative motion in the visual system of the fly II. Figure-detection cells, a new class of visual interneurons. Biol Cybern 52: 195-209

Egelhaaf M (1987) Dynamic properties of two control systems underlying visually guided turning in house-flies. J Comp Physiol A 161: 777-783

Egelhaaf M, Borst A (1989) Transient and steady-state response properties of movement detectors. J Opt Soc Am A 6: 116-127 and erratum 7: 172

Egelhaaf M, Borst A (1993) A look into the cockpit of the fly: visual orientation, algorithms, and identified neurons. J Neurosci 13: 4563-4574

Egelhaaf M, Hausen K, Reichardt W, Wehrhahn C (1988) Visual course control in flies relies on neuronal computation of object and background motion. Trends Neurosci 11: 351-358

Egelhaaf M, Borst A, Warzecha A-K, Flecks S, Wildemann A (1993) Neural circuit tuning fly visual interneurons to motion of small objects. II. Input organization of inhibitory circuit elements revealed by electrophysiological and optical recording techniques. J Neurophysiol 69: 340-351

Fermi G, Reichardt W (1963) Optomotorische Reaktionen der Fliege Musca domestica. Kybernetik 2: 15-28

Frost BJ, Nakayama K (1983) Single visual neurons code opposing motion independent of direction. Science 220: 744-745

Frost BJ, Cavanagh P, Morgan B (1988) Deep tectal cells in pigeons respond to kinematograms. J Comp Physiol A 162: 639647

Götz KG (1964) Optomotorische Untersuchungen des visuellen Systems einiger Augenmutanten der Fruchtfliege Drosophila. Kybernetik 2: 77-92

Götz KG (1968) Flight control in Drosophila by visual perception of motion. Kybernetik 4: 199-208

Hausen K (1982a) Motion sensitive interneurons in the optomotor system of the fly. I. The horizontal cells: structure and signals. Biol Cybern 45: 143-156 
Hausen K (1982b) Motion sensitive interneurons in the optomotor system of the fly. II. The horizontal cells: receptive field organization and response characteristics. Biol Cybern 46: 67-79

Helmholtz Hv (1866) Handbuch der physiologischen Optik. Voss Verlag, Hamburg

Hengstenberg R (1982) Common visual response properties of giant vertical cells in the lobula plate of the blowfly Calliphora. J Comp Physiol A 149: 179-193

Kern R (1994) Mechanisms underlying visual position stabilization in the hovering hawk moth Macroglossum stellatarum L. Doctoral thesis, Universität Tübingen

Kimmerle B, Egelhaaf M, Srinivasan MV (1996) Object detection by relative motion in freely flying flies. Naturwissenschaften 83 : 380-381

Land MF, Collett TS (1974) Chasing behaviour in houseflies (Fannia canicularis). A description and analysis. J Comp Physiol A 89: $331-357$

Lehrer M, Srinivasan MV (1993) Object detection by honeybees: why do they land on edges? J Comp Physiol 173: 23-32

Maddess T, Laughlin SB (1985) Adaptation of the motion-sensitive neuron $\mathrm{H} 1$ is generated locally and governed by contrast frequency. Proc R Soc Lond B 225: 251-275

Miles FA, Kawano K (1987) Visual stabilization of the eyes. Trends Neurosci 10: 153-158

Pfaff M, Varjú D (1991) Mechanisms of visual distance perception in the hawk moth Macroglossum stellatarum. Zool Jb Physiol 95: 315-321

Reichardt W, Poggio T (1979) Figure-ground discrimination by relative movement in the visual system of the fly. Part I: Experimental results. Biol Cybern 35: 81-100
Reichardt W, Poggio T, Hausen K (1983) Figure-ground discrimination by relative movement in the visual system of the fly. Part II: towards the neural circuitry. Biol Cybern 46 [Suppl]: $1-30$

Srinivasan (1993) How insects infer range from visual motion. In: Miles FA, Wallman $\mathbf{J}$ (eds) Visual motion and its role in the stabilization of gaze. Elsevier, Amsterdam pp 139-156

Srinivasan MV, Lehrer M, Horridge GA (1990) Visual figureground discrimination in the honeybee: the role of motion parallax at boundaries. Proc R Soc Lond B 238: 331-350

Sterling P, Wickelgren BG (1969) Visual receptive fields in the superior colliculus of the cat. J Neurophysiol 32: 1-15

Tanaka K, Hikosaka K, Saito H, Yukie M, Fukada Y, Iwai E (1986) Analysis of local and wide-field movements in the superior temporal visual areas of the macaque monkey. J Neurosci 6: 134-144

Virsik RP, Reichardt W (1976) Detection and tracking of moving objects by the fly Musca domestica. Biol Cybern 23: 83-98

Wagner H (1986) Flight performance and visual control of flight of the free-flying housefly (Musca domestica L.) II. Pursuit of targets. Philos Trans R Soc Lond B 312: 553-579

Warzecha A-K, Egelhaaf M, Borst A (1993) Neural circuit tuning fly visual interneurons to motion of small objects. I. Dissection of the circuit by pharmacological and photoinactivation techniques. J Neurophysiol 69: 329-339

Wehrhahn C (1979) Sex-specific differences in the chasing behaviour of houseflies (Musca). Biol Cybern 32: 239-241

Xiao D-K, Raiguel S, Marcar V, Koenderink JJ, Orban A (1995) Spatial heterogeneity of inhibitory surrounds in the middle temporal visual area. Proc Natl Acad Sci USA 92: 11303-11306 